\title{
Magnetohydrodynamic accretion flows around compact objects
}

\author{
Santabrata Das* \\ ARCSEC, 98 Gunja-Dong, Gwangjin-Gu, \\ Seoul 143-747, Sejong University, South Korea. \\ E-mail: sbdas@canopus.cnu.ac.kr
}

\section{Sandip K. Chakrabarti}

S. N. Bose National Centre for Basic Sciences, Saltlake, Kolkata, India

E-mail: chakraba@bose.res.in

\begin{abstract}
Matter accreting onto compact objects should be magnetized. We solve the magnetohydrodynamic equations governing axisymmetric flows around compact objects and present all possible solution topologies for the accretion flow. We divide the parameter space spanned by the energy and angular momentum of the flow in terms of the flow topologies. We separate the region of parameter space which produce standing magnetohydrodynamic shock waves in the accretion flow. We also show regions of the parameter space where the shocks may oscillate. In the astrophysical context, these solutions are very important as they could give rise to Quasi-Periodic Oscillations seen in hard $\mathrm{X}$ rays.
\end{abstract}

VI Microquasar Workshop: Microquasars and Beyond

September 18-22 2006

Società del Casino, Como, Italy

\footnotetext{
${ }^{*}$ Speaker.
} 


\section{Introduction}

Magnetic field is ubiquitous in nature and indeed, in many astrophysical circumstances it is dynamically important. After the earlier attempts of Mestel (1967) and Weber \& Davis (1967) for solar winds, Chakrabarti (1990) generalized these works for rotating compact stars and black holes and found a few solution topologies which are relevant to accretion and winds. Takahashi et al. (1990) and his group solved the MHD equations in Kerr geometry while looking for possible ways to extract energies from black holes. They discussed the occurrences of the sonic points. Nitta et al. (1991) solved the Grad-Safranov equation and found a few of the global topologies. However, so far, the study of solution topologies and their dependence on the flow parameters was not explored. In the present paper, we are focusing our attention to study the trans-magnetosonic flow properties in accretion around compact objects in presence of both radial and toroidal magnetic fields. We explore the dependence of the solution topology on the parameter space spanned by the energy and angular momentum of the flow. For a given pair of Alfven radius and velocity, we could identify as many as eighteen types of solution topologies and divide the parameter space according to the nature of the solution. We also study the properties of the standing magneto-hydrodynamic shock waves. Sometimes, a high energy spectrum is observed up to $\sim 20 \mathrm{MeV}$ which could be achieved through shock acceleration of electrons. Thus, it is important to look for solutions which will include standing or propagating shocks. These accelerated charged particles will produce powerlaw synchrotron radiations which could be an important tool in explaining the spectra of black hole candidates. In addition, at the shock (in the so-called magnetized CENBOL region), soft photons are energized through inverse Comptonization by the hot electrons. In this paper, we concentrate on the solutions of the magnetohydrodynamic (MHD) problem which include standing shocks and classify the parameter space which allow shocks in the accretion flows.

\section{Governing equations and Magnetosonic point analysis}

We start with a stationary, axisymmetric, thin, non-self-gravitating, non-dissipative, highly conducting, wedge-shaped, adiabatic flow on the equatorial plane of a compact star which is described by the Paczyński-Wiita (1980) potential. We use the flow model to be the same as that of Chakrabarti (1990) and Das \& Chakrabarti (2006). Magnetohydrodynamic flow equations on the equatorial plane are as follows: (a) The energy conservation equation: $E=\frac{1}{2} \vartheta_{r}^{2}+\frac{1}{2} \vartheta_{\phi}^{2}+$ $\frac{\gamma}{\gamma-1} \frac{p}{\rho}+\Phi(r)-\frac{B_{\phi} B_{r} \Omega r}{4 \pi \rho \vartheta_{r}}$, (b) The angular momentum conservation equation, $L=r \vartheta_{\phi}-\frac{B_{\phi} B_{r} r}{4 \pi \rho \vartheta_{r}}$, (c) The mass flux conservation equation, $\dot{M}=\rho \vartheta_{r} r^{2}$, (d) The radial magnetic-flux conservation equation, $B_{r} r^{2}=C_{1}$, and, (e) Maxwell's equation $(\mathbf{E}=-\vartheta \times \mathbf{B}=\mathbf{0})$ for a perfectly conducting fluid on the neutron star surface: $r\left(\vartheta_{r} B_{\phi}-\vartheta_{\phi} B_{r}\right)=-\Omega C_{1}$, where, we assumed that on the surface $r=r_{n}, \vartheta_{r}=0$. In case of a non-rotating black hole, where $\vartheta_{\phi}=0$ on $r=r_{g}$, the horizon, the above condition becomes, $r\left(\vartheta_{r} B_{\phi}-\vartheta_{\phi} B_{r}\right)=r_{g} \vartheta_{r g} B_{\phi g}=A$ (constant). Here, $\vartheta_{r}$ and $\vartheta_{\phi}$ are the radial and azimuthal components of velocity, $B_{r}$ and $B_{\phi}$ are the radial and azimuthal components of magnetic field, $\phi(r)=-\frac{G M}{\left(r-2 G M / c^{2}\right)}$ is the gravitational potential due to the compact object and $\Omega$ is the constant angular velocity of the neutron star or the black hole. Furthermore, $P, \rho$ and $\gamma$ being the pressure, density and adiabatic index respectively. We assume the $B_{\theta}$ component to be negligible since the flow is considered to be sufficiently thin. We rewrite the energy expression as, 
$\mathscr{E}=E-L \Omega=\frac{1}{2} \vartheta_{r}^{2}+n a^{2}+\frac{1}{2} \frac{\left(\vartheta_{r}-1\right)^{2} \Omega^{2} r^{2}}{\left(\vartheta_{r} r^{2}-1\right)^{2}}+\Phi(r)-\frac{\left(\vartheta_{r}-1\right) \Omega^{2} r^{2}}{\vartheta_{r} r^{2}-1}$. In this study, lengths will be measured in units of the Alfvén radius $r_{a}$, velocities will be measured in units of the Alfvén speed $\vartheta_{a}$ and time will be measured in units of $r_{a} / \vartheta_{a}$. In this case, $L=\Omega$.

In order to study the magnetohydrodynamics flow properties we first calculate the radial velocity gradient $\left(d \vartheta_{r} / d r\right)$ by simplifying governing equations which is obtained as (Chakrabarti, 1990), $\frac{d \vartheta_{r}}{d r}=\frac{N}{D}$, where, the numerator $N$ is given by, $N=\vartheta_{r} r\left[\left(\vartheta_{r} r^{2}-1\right)^{2}\left(\vartheta_{r}-1\right) \Omega^{2}+\left(\frac{2 a^{2}}{r^{2}}-\right.\right.$ $\left.\left.\frac{1}{r} \frac{d \Phi}{d r}\right)\left(\vartheta_{r} r^{2}-1\right)^{3}+\vartheta_{r} \Omega^{2}\left(1-r^{2}\right)\left(\vartheta_{r}-1\right)\left(1+\vartheta_{r} r^{2}\right)\right]$ and the denominator $D$ is given by, $D=$ $\left(\vartheta_{r}^{2}-a^{2}\right)\left(\vartheta_{r} r^{2}-1\right)^{3}-\left(r^{2}-1\right)^{2} \vartheta_{r}^{2} r^{2} \Omega^{2}$. It is clear that both the numerator and denominator simultaneously become zero at the Alfvén point $\left(r=1, \vartheta_{r}=1\right)$. Apart from the Alfvén point, they may vanish at some other points also. These points are called the critical points and are commonly known as the magnetosonic points. At these points, the flow velocity becomes equal to the speed of the magnetosonic waves. At the outer edge of the accretion disk, the radial velocity of the flow remains sub-magnetosonic. For neutron star accretion, matter accretes on to the star surface submagnetosonically. Thus the flow may or may not become super fast- or slow-magnetosonic before touching the star surface. For a black hole accretion, however, the inner boundary condition is different but unique. Here the flow must cross the horizon super-magnetosonically, i.e., the flow must cross the magnetosonic point at least once before entering into the black hole.

\section{Behaviour of parameter space, solution topologies and MHD shocks}

In Fig. 1 (top-left), we divide the parameter space spanned by the energy and angular momentum of the flow into various regions depending on the nature of the solution topologies. Accretion flow solutions in black hole and neutron stars differ only through inner boundary conditions. For a pair of flow parameters, the curves representing the solutions topologies remain the same, and only depend on the flow parameters. For each topology we need to put a boundary condition to decide whether the solution is for black hole or for a neutron star. The curve ADE is obtained for the special case where the radial velocity $\vartheta_{r}=0$ at all the sonic points. Below the curve $A D E$, no solution is possible. The curve PG is obtained by considering the flow velocity at the magnetosonic point (occurring at $r<1$ ) is exactly same as the Alfvén velocity. Other curves are obtained following Chakrabarti(1990) where the magnetosonic points with (a) $r<1, \vartheta_{r}<1$, (b) $r<1, \vartheta_{r}>1$, (c) $r>1, \vartheta_{r}<1$ and (d) $r>1, \vartheta_{r}>1$ are denoted as 'Bondi-like Slow', 'Bondi-like Fast', 'Rotational Slow' and 'Rotational Fast' respectively. The Bondi-like slow magnetosonic points exist in a region surrounded by the boundary PBEGHP. The Rotational-slow magnetosonic points exist in a region surrounded by the boundary ABCFGUA (for $L>0$ ). The Rotational-fast magnetosonic points exist in a region surrounded by the boundary RDEQR and $L=0$ and $\mathscr{E}>0$. The Bondi-like fast magnetosonic points exist in a region surrounded by the boundary QGUQ (for $L>0$ ). The region surrounded by the boundary SKTS and shaded by the dots indicates the region in which magnetosonic shocks may form. The region of the parameter space shaded by the dot-dash lines denotes the forbidden region for the flow solution. The region surrounded by the boundary PCKOTUQP has the solutions topologies to form magnetosonic shocks, but the standing shocks do not form as the shock conditions are not satisfied.

In order to form a shock, the accretion flow must have at least two magnetosonic points since the inner boundary condition is that a black hole accretion is necessarily super-magnetosonic on 

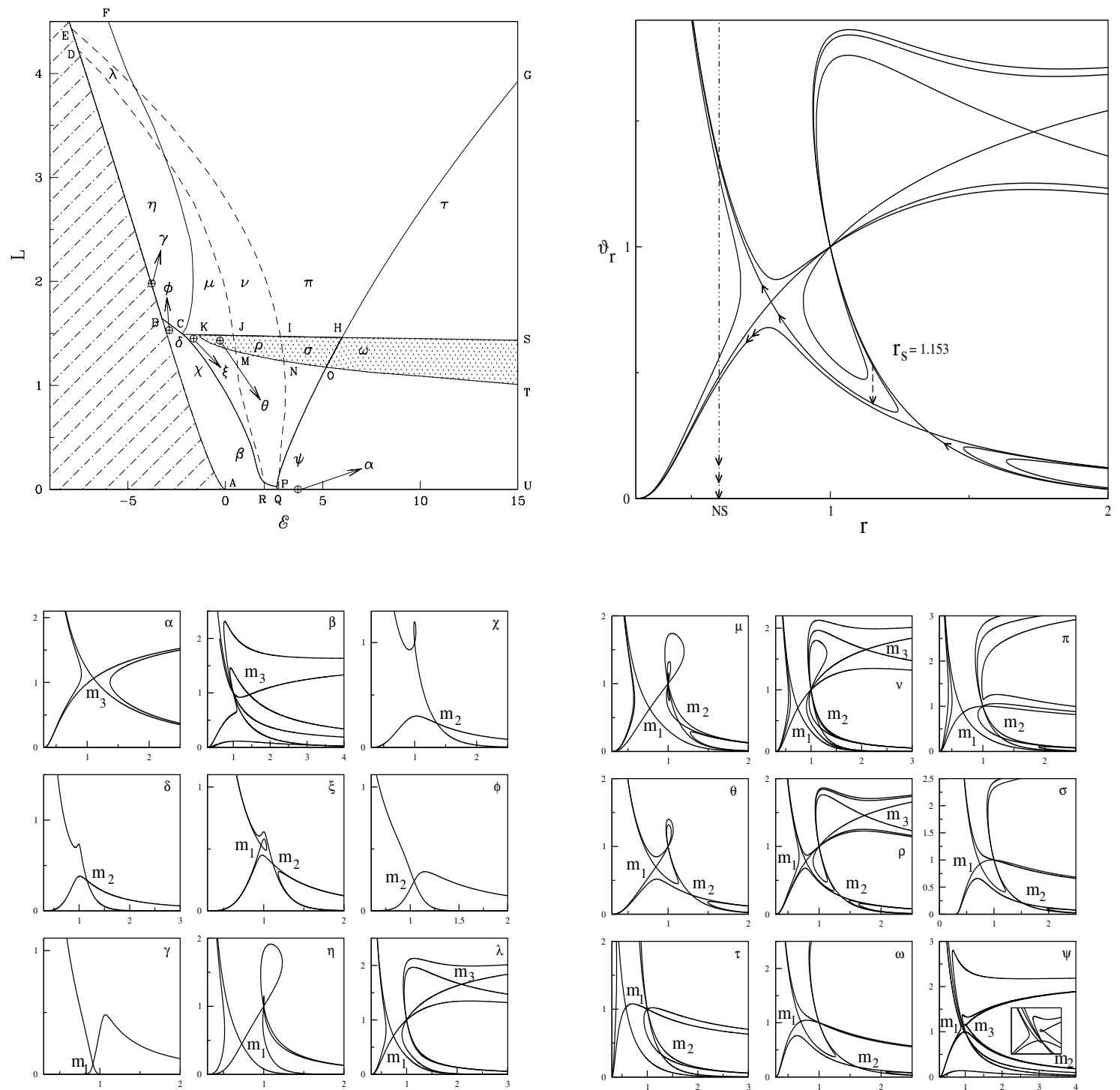

Fig. 1: (Top-left:) Division of parameter space according to solution topologies.(Top-right:) Example of complete solution topology with standing magnetohydrodynamic shock waves. (Bottom-left \& right:) Solutions of the magneto-hydrodynamic flows for various $(\mathscr{E}, L)$ pairs of conserved parameters. Greek alphabets mark different types of solutions drawn with parameters from different regions marked in Top-left figure. Along horizontal axis is the radial distance and along vertical axis is the radial velocity.

the horizon and the shock conditions have to be satisfied in between these two points. Here the flow, after crossing the rotational-slow magnetosonic point, will pass through a (slow) shock and subsequently pass through a Bondi-like slow magnetosonic point provided the entropy at the inner magnetosonic point is higher compared to that at the outer magnetosonic point. The difference in entropy is guaranteed to be generated at the shock (through turbulence, for instance) if the shock conditions are fulfilled. In Fig. 1 (bottom-right), we present an example of the shock solution, obtained using parameters from the region SKTS, which possesses standing, slow-magnetosonic shock. We chose Alfvén velocity and radius as $\vartheta_{a}=10^{10} \mathrm{~cm} \mathrm{~s}^{-1}$ and $r_{a}=10^{7} \mathrm{~cm}$ respectively 
while the central mass was chosen to be $10 M_{\odot}$ for illustration purpose. The parameter $(\mathscr{E}, L)$ pair is $(1.5,1.45)$ and the shock locations is $r_{s}=1.153$. The standing shock locations is also marked with the vertical dashed lines. The single arrowed curve represents the solution towards a black hole. The double-arrowed curve, which is sub-Alfvénic throughout the flow is appropriate for a neutron star accretion without a shock transition. The triple-arrowed vertical line (dash-dotted) indicates a typical shock transition at the boundary of the neutron star. The post-shock region where radial flow is slowed down and flow becomes hotter, is the most important region as this is the place where hard $\mathrm{X}$ rays are generated.

The entire parameter space is sub-divided further according to the nature of the solutions. We scanned the complete parameter space and obtained eighteen distinct types of solutions as shown in Fig. 1 (top-left and bottom-right). Each solution type is identified by a Greek alphabet $\alpha, \beta, .$. etc. These Greek alphabets in the parameter space denote the regions of the parameters for which the solutions are drawn. In Fig. 1(bottom-left \& right), the Bondi-like (slow/fast), Rotational slow and Rotational fast magnetosonic points are denoted by $m_{1}, m_{2}$, and $m_{3}$ respectively. For $L=0$, since $\vartheta_{\phi}=B_{\phi}=0$, the solutions become identical to Bondi type.

\section{Concluding Remarks}

We presented all possible solution topologies of a magnetohydrodynamic, quasi-equatorial, adiabatic flow around compact objects and divided the entire parameter space in terms of different types of the flow topologies. We show that in a significant region the flow satisfies the standing shock conditions while in another region shock conditions are not satisfied but as the inner magnetosonic points have higher entropy than the outer magnetosonic points, the flow is likely to pass through a shock which oscillates as in the case of the hydrodynamic flow. The post-shock region inverse Comptonizes soft photons from the pre-shock flow either from the Keplerian disk or from the synchrotron radiation and re-emits them as hard X-rays (Chakrabarti \& Mandal, 2006). Since MHD shocks occur closer to the black hole, they give rise to the high frequency quasi-periodic oscillations of hard $\mathrm{X}$ rays as observed in several black hole candidates.

Acknowledgments: This work is partly supported by a project (Grant No. SP/S2/K-15/2001) funded by DST. India. SD is thankful for financial support to KOSEF through ARCSEC, Korea.

\section{References}

[1] Mestel, L., 1967, Plasma Astrophysics, ed. Sturrock, P. A., Academic Press, New York

[2] Weber, E. J. \& Davis, L. Jr., 1967, ApJ 148, 217

[3] Chakrabarti, S. K., 1990, MNRAS 246, 134

[4] Chakrabarti, S. K. and S. Mandal, 2006, ApJ, 642, L49

[5] Takahashi, M., Nitta, S., Tatematsu, Y. \& Tomimatsu, A., 1990, ApJ 363, 206

[6] Nitta, S., Takahashi, M. \& Tomimatsu, A., 1991, Phys. Rev. D 44, 2295

[7] Paczyński B., Wiita P.J., 1980, A \& A 88, 23

[8] Das, S., Chakrabarti, S. K., 2006, accepted in MNRAS. 